\title{
Simplified Ordering for Fixed-complexity Sphere Decoder
}

\author{
X. G. Dai \\ Department of Electrical \& \\ Electronic Engineering \\ The University of Hong Kong \\ xgdai@eee.hku.hk
}

\author{
S. W. Cheung \\ Department of Electrical \& \\ Electronic Engineering \\ The University of Hong Kong \\ swcheung@eee.hku.hk
}

\author{
T. I. Yuk \\ Department of Electrical \& \\ Electronic Engineering \\ The University of Hong Kong \\ tiyuk@eee.hku.hk
}

\begin{abstract}
This paper proposes a simplified ordering algorithm for the fixedcomplexity sphere decoder (FSD). The new algorithm is developed from the analysis of the ordering for FSD from a geometrical point of view. Computer simulation is used to assess the improvements in bit-error rate (BER) performances of MIMO systems using the FSD with the original and the simplified ordering. Simulation results show that the new ordering method can achieve nearly the same BER as the original ordering method but with much less complexity.
\end{abstract}

\section{Categories and Subject Descriptors \\ C.2 [Computer-Communication Networks]: Wireless Communication.}

\section{General Terms}

Algorithms, Design.

\section{Keywords}

geometrical analysis, fixed-complexity sphere decoder, ordering, MIMO.

\section{INTRODUCTION}

Multiple-input-multiple-output (MIMO) system has attracted more and more attention in wireless communications due to its potentially high data rate and low bit-error rate (BER). However, detection has been a big issue that makes MIMO systems difficult to implement in practice. It is well known that maximumlikelihood (ML) detection achieves the best block-error rate (BLER) performance, but its complexity is too high because it has to search the solution in the whole signal space of the transmitted signals. Linear detection algorithms such as zero-forcing (ZF) and minimum mean-square-error (MMSE) are much simpler, but the BER performance is not satisfactory because they do not fully explore the diversity advantages of the MIMO channel. Sphere decoding (SD) [1]-[3] can achieve the BER performance of ML detection with much less complexity by searching within only a subset of the whole signal space. Geometric decoding algorithms [4]-[6] have also been proposed to reduce the search signal space to a small subset. However, these algorithms share a common disadvantage that the detection complexity varies with the signalto-noise ratio (SNR), making these algorithms difficult for hardware implementation.

Permission to make digital or hard copies of all or part of this work for personal or classroom use is granted without fee provided that copies are not made or distributed for profit or commercial advantage and that copies bear this notice and the full citation on the first page. To copy otherwise, or republish, to post on servers or to redistribute to lists, requires prior specific permission and/or a fee. "IWCMC'10, June 28July 2, 2010, Caen, France. Copyright (C) 2010 ACM 978-1-4503-00629/10/06/...\$10.00"
Recently, a fixed-complexity sphere decoder (FSD) has been proposed to solve the variable complexity problem of SD [7]. The algorithm used an ordering process to determine the order of symbols to be detected and then enumerated different numbers of candidates for each symbol. Then it calculated the Euclidean distances between the received signal and all the vector combinations of these candidates and chose the vector with the smallest Euclidean distance as the detected symbol. Since the FSD searches for the candidates in a processive way rather than a recursive way as in $\mathrm{SD}$, the detection complexity is fixed for all SNRs.

The error performance of FSD was analyzed in [8] algebraically. In this paper, we look at FSD from a geometrical point of view. We show how the ordering criterion in FSD works in the lattice of the receive signal space. Then we adopt a simplified ordering criterion to approximate the original ordering criterion for FSD. Simulation results show that, in a $4 \times 4$ MIMO system used for study, the simplified ordering achieves nearly the same BER performance as the original ordering for FSD with much less complexity.

The rest of this paper is organized as follows: Section II describes the system model and the FSD algorithm in [7]. Section III explains the geometrical analysis of the ordering used in FSD. Section IV presents the simplified ordering algorithm based on the analysis in Section III. Simulation results are presented in Section V. Section VI concludes the paper.

\section{SYSTEM MODEL AND FIXED- COMPLEXITY SPHERE DECODER}

\subsection{System Model}

The system model used for the study is an $N_{\mathrm{t}} \times N_{\mathrm{r}}$ MIMO system with $N_{\mathrm{t}}$ transmit antennas and $N_{\mathrm{r}}$ receive antennas, over a fast Rayleigh fading channel which changes at each transmitted symbol vector. The received signal vector $\mathbf{r}$ with length $N_{\mathrm{r}}$ is given by:

$$
\mathbf{r}=\mathbf{H s}+\mathbf{w}
$$

where the entries of $\mathbf{H} \in \mathbb{C}^{N_{r} \times N_{t}}$ represent the channel coefficients which are assumed to be perfectly known at the receiver but not at the transmitter, $\mathbf{s} \in \mathbb{C}^{N_{t}}$ is the transmitted uncoded signal vector, and $\mathbf{w} \in \mathbb{C}^{N_{r}}$ represents the complex additive white Gaussian noise (AWGN) matrix with elements being independently and identically distributed (iid) and following the normal distribution $N_{\mathrm{C}}\left(0, N_{0}\right)$. 
The detection process aims to find the signal vector with the smallest Euclidean distance from the received vector:

$$
\hat{\mathbf{s}}=\arg \min _{\mathbf{s} \in \mathbb{S}}\|\mathbf{r}-\mathbf{H s}\|^{2}
$$

where $\mathbb{S}$ is the signal space containing all the possible combinations of transmitted symbols.

\subsection{FSD algorithm}

In [7], the FSD algorithm tried to solve (2) in two steps. First, an ordering was used to determine the order of symbols to be detected. For the first $p$ symbols, all or some of the possible values were enumerated (called full or partial expansion). An SDlike detection algorithm based on the Cholesky decomposition of the channel matrix was then used to detect the rest of the symbols. Finally, the Euclidean distances between all the enumerated symbol vectors and the received signal $\mathbf{r}$ were calculated and compared. The symbol vector with the smallest Euclidean distance from $\mathbf{r}$ was taken as the detected vector. These two steps can be described in details as follows.

\section{a. Ordering}

The channel matrix ordering of [7] can be described as follows.

In the $(i+1)^{\text {th }}$ iteration, for $i=0, \ldots, N_{\mathrm{t}}-1$ :

1) The matrix $\mathbf{Q}_{i}=\left(\mathbf{H}_{N_{t}-i}^{H} \mathbf{H}_{N_{t}-i}\right)^{-1}$ is calculated, where $\mathbf{H}_{N_{t}-i}$ is the channel matrix with the $i$ columns selected in the previous iterations removed and $\mathbf{H}_{N_{t}-i}^{H}$ denotes the conjugate transposition of $\mathbf{H}_{N_{t}-i}$.

2) The $k^{\text {th }}$ column of $\mathbf{H}_{N_{t}-i}$ is selected according to

$$
k= \begin{cases}\arg \max _{j}\left[\mathbf{Q}_{i}\right]_{j j}, & \text { for full expansion, } \\ \arg \min _{j}\left[\mathbf{Q}_{i}\right]_{j j}, & \text { for single expansion, }\end{cases}
$$

where $\left[\mathbf{Q}_{i}\right]_{j j}$ is the $j^{\text {th }}$-diagonal element of $\mathbf{Q}_{i}$. In [8], $\left[\mathbf{Q}_{i}\right]_{j j}$ was interpreted as the post-processing noise amplification of the $j^{\text {th }}$ symbol in the $(i+1)^{\text {th }}$ iteration. In the next section, we show what $\left[\mathbf{Q}_{i}\right]_{j j}$ is in the geometrical expression of FSD detection.

\section{b. Detection}

After ordering, expansions and detections are used. As an example, consider a system transmitting four 16QAM symbols (i.e. $N_{\mathrm{t}}=4$ ) at a time. To detect the first symbol (e.g. $s_{2}$ after ordering), full expansion is used (i.e. $p=1$ ). For each of the 16 possible values of $s_{2}$, the contribution of $s_{2}$ is removed from the received signal $\mathbf{r}$ and hard decision is applied to detect the next symbol by minimizing the partial Euclidean distance (PED) [9]. Interference in the detected symbol is then removed from $\mathbf{r}$ and similarly, the PED of the next level is minimized to detect the next symbol. The detection process goes like this until all the 16 symbol-vector candidates are obtained for each value of $s_{2}$. The Euclidean distances of these 16 candidates to the received signal $\mathbf{r}$ are compared. The candidate with the smallest Euclidean distance is taken as the detected vector.

\section{GEOMETRICAL ANALYSIS OF FSD ORDERING}

In this section, we illustrate the ordering for FSD from a geometrical point of view.

Consider an example of a 3-dimension real received signal space (i.e. $N_{\mathrm{r}}=N_{\mathrm{t}}=3$ ) for $4 \mathrm{PAM}$ as shown in Fig. 1, where the symbol $\star$ denotes the received signal vector $\mathbf{r}$. The generation matrix of the lattice is $\mathbf{H}$, having column vectors $\mathbf{h}_{1}, \mathbf{h}_{2}$ and $\mathbf{h}_{3}$ as the directions of $s_{1}, s_{2}$ and $s_{3}$, respectively. The detector aims to find the lattice point closest to $\mathbf{r}$. In Fig. 1, the dots of the same color denote the lattice points with the same values of $s_{2}$. We call the hyperplane formed by the dots with the same value of $s_{2}$ a layer of $s_{2}$. For example, the layers with slashes are layers of $s_{3}$.

In Section II, it is described that, in each iteration of the ordering process, one column of $\mathbf{H}$ is removed. Therefore, in the $(i+1)^{\mathrm{th}}$ iteration, for $i=0,1, \ldots, N_{\mathrm{t}}-1$, the space is spanned by only the columns of $\mathbf{H}_{N_{t}-i}$ and the lattice is generated by $\mathbf{H}_{N_{t}-i}$. We denote the index of the symbol corresponding to the $j^{\text {th }}$ column of $\mathbf{H}_{N_{t}-i}$ by $I(i, j)$, and the distance between the layers of $s_{I(i, j)}$ in the $(i+1)^{\text {th }}$ iteration by $l_{j}^{i}$ (for $j=1,2, \ldots, N_{\mathrm{t}}$ ). Then in the $1^{\text {st }}$ iteration $(i=0)$ we have the following equation.

$$
l_{j}^{0}=\frac{2}{\sqrt{\left[\mathbf{Q}_{0}\right]_{j j}}}
$$

Proof: In this proof, we use the 3-dimensional example of Fig. 1. The extension to an $N_{\mathrm{t}}$-dimensional space is straightforward.

Denoting the matrix $\left(\mathbf{H}_{0}^{H} \mathbf{H}_{0}\right)^{-1} \mathbf{H}_{0}^{H}$ as $\mathbf{M}_{z f}$, we have

$$
\mathbf{M}_{z f} \mathbf{H}_{0}=\mathbf{I}
$$

Then denoting the $j^{\text {th }}$ row of $\mathbf{M}_{z f}$ as $\mathbf{m}_{j}^{H}$, (5) implies $\mathbf{m}_{j}$ is orthogonal to all but the $j^{\text {th }}$ column vectors of $\mathbf{H}_{0}$. Also note that

$$
\mathbf{m}_{j}=\mathbf{H}_{0} \mathbf{q}_{j}
$$

where $\mathbf{q}_{j}^{H}$ is the $j^{\text {th }}$ row of $\mathbf{Q}_{0}$. (6) means that $\mathbf{m}_{j}$ is within the vector space spanned by the columns of $\mathbf{H}_{0}$. From (5) and (6), we can see that $\mathbf{m}_{j}$ is orthogonal to the layers of $s_{j}$. The length of the line segment of $\mathbf{m}_{j}$ between adjacent layers of $s_{j}$ is equal to the distance between adjacent layers of $s_{j}$, which is $l_{j}^{0}$. We denote $\mathbf{h}_{j}^{0}$ as the $j^{\text {th }}$ column of $\mathbf{H}_{0}$ and || as the Euclidean norm of a vector. Then, from Fig. 1, we can see that the length of the line segment of $\mathbf{h}_{j}^{0}$ between adjacent layers of $s_{j}$ is equal to $2\left|\mathbf{h}_{j}^{0}\right|$. Since the layers of $s_{j}$ are parallel to each other, we have:

$$
l_{j}^{0}=2\left|\mathbf{h}_{j}^{0}\right| \cos \theta
$$

where $\theta$ is the angle between $\mathbf{m}_{j}$ and $\mathbf{h}_{j}^{0}$.

From (5) we can write: 


$$
\mathbf{m}_{j}^{H} \mathbf{h}_{j}^{0}=\left|\mathbf{m}_{j}\right|\left|\mathbf{h}_{j}^{0}\right| \cos \theta=1
$$

Combining (7) and (8) yields:

$$
l_{j}^{0}=\frac{2}{\left|\mathbf{m}_{j}\right|}
$$

From (6) we know

$$
\left|\mathbf{m}_{j}\right|^{2}=\mathbf{q}_{j}^{H} \mathbf{H}_{0}^{H} \mathbf{H}_{0} \mathbf{q}_{j}
$$

Since $\mathbf{q}_{j}^{H}$ is the $j^{\text {th }}$ row of $\mathbf{Q}_{0}$ and $\mathbf{Q}_{0} \mathbf{H}_{0}^{H} \mathbf{H}_{0}=\mathbf{I}, \mathbf{q}_{j}^{H} \mathbf{H}_{0}^{H} \mathbf{H}_{0} \mathbf{q}_{j}$ is equal to the $j^{\text {th }}$ element of $\mathbf{q}_{j}$, which is $\left[\mathbf{Q}_{0}\right]_{j j}$. Substituting $\mathbf{q}_{j}^{H} \mathbf{H}_{0}^{H} \mathbf{H}_{0} \mathbf{q}_{j}$ into (10) and then to (9) yields

$$
l_{j}^{0}=\frac{2}{\sqrt{\left[\mathbf{Q}_{0}\right]_{j j}}}
$$

Similarly, it can be proved that in the $(i+1)^{\text {th }}$ iteration:

$$
l_{j}^{i}=\frac{2}{\sqrt{\left[\mathbf{Q}_{i}\right]_{j j}}}
$$

From the previous analysis, we can see that the ordering for FSD actually depends on the distance between the layers of the symbols. The $p$ symbols with the smallest layer distances are fully expanded. In single expansion, the symbol with a larger layer distance is detected first.

To understand the reason for this ordering used in FSD, we can look at it from a geometrical way. Consider the same lattice shown in Fig.1. The layer distance of $s_{2}$ is obviously smaller than those of $s_{1}$ and $s_{3}$, so the lattice point closest to the received point $\mathbf{r}$ (the $\star$ ) is equally likely to be in one of the four layers of $s_{2}$. Thus all the four layers of $s_{2}$ need to be searched and the full expansion process is used in FSD. In single expansion, the $2^{\text {nd }}$ column of $\mathbf{H}$ is removed and the space becomes a 2-dimensional space spanned by $\mathbf{h}_{1}$ and $\mathbf{h}_{3}$, as depicted in Fig. 2. Now the symbols are detected iteratively and only one candidate of each symbol is taken. If the layer distance of a symbol is large, it is likely that the lattice point closest to $\mathbf{r}$ is on the layer closest to $\mathbf{r}$. In the example of Fig. 2, the distance between the layers of $s_{3}$ is large, so the point closest to $\mathbf{r}$ is more likely to be on the layer where $s_{3}=-1$, which is the closest layer to $\mathbf{r}$. This means that the decision of $s_{3}=-1$ has a higher probability of being correct, so detecting $s_{3}$ first will reduce the error propagation in single expansion detection. This is the reason why the symbols corresponding to small $\left[\mathbf{Q}_{i}\right]_{j j}$ are detected first in single expansion detection in FSD.

\section{SIMPLIFIED ORDERING}

As we can see from section II, the ordering for FSD requires at least $N_{\mathrm{t}}-1$ matrix inversion operations, which contributes to much of decoding complexity. This problem is even worse for a fast fading channel where the channel matrix changes at each transmitted symbol vector. In this section, we propose a simplified ordering criterion based on the geometrical analysis to approximate the original ordering criterion for FSD. This new ordering requires only one matrix inversion operation and thus reduces the complexity of the ordering process for FSD. The simplified ordering is described as follows:

The $k^{\text {th }}$ symbol is selected according to

$$
k= \begin{cases}\arg \max _{j}\left[\mathbf{Q}_{0}\right]_{j j}, & \text { for full expansion, } \\ \arg \min _{j}\left[\mathbf{Q}_{0}\right]_{j j}, & \text { for single expansion, }\end{cases}
$$

where $\left[\mathbf{Q}_{0}\right]_{j j}$ is the $j^{\text {th }}$-diagonal element of $\mathbf{Q}_{0}$ and $\mathbf{Q}_{0}=\left(\mathbf{H}^{H} \mathbf{H}\right)^{-1}$.

In the example of Fig. 1 and Fig. 2, the simplified ordering is equivalent to using $l_{3}^{0}$ to approximate $l_{2}^{1}$. This approximation is more clearly depicted in Fig. 3. The extension to an $N_{\mathrm{t}^{-}}$ dimensional system is straightforward. Recall the definition of $I(i, j)$ in the last section. It is easy to see that in the $i^{\text {th }}$ iteration (for $i=0,1, \ldots, N_{\mathrm{t}}-1$ ) of ordering in an $N_{\mathrm{t}}$-dimensional system, the symbol corresponding to $l_{j}^{i}$ is $s_{I(i, j)}$ and $I(0, j)=j$. Thus a simplified method is to use $l_{I(i, j)}^{0}$ to approximate $l_{j}^{i}$. As we can see from the example of Fig. 3, this approximation uses the layer distance in a 3-dimensional space to approximate the layer distances in a 2-dimensional space. Generally, it uses an $N_{\mathrm{t}^{-}}$ dimensional layer distance to replace all the layer distances in the lower dimensional spaces. Although errors may occur in approximating the distances, the ordering is less affected. Moreover, the simplified ordering of (13) only requires one matrix inversion operation, which substantially reduces the complexity of the ordering, especially in the fast fading channels.

\section{SIMULATION RESULTS}

Computer simulation has been used to evaluate the effects of ordering and simplified ordering on the BER performance and the complexity of a MIMO system. A $4 \times 4$ MIMO system transmitting 4 uncoded 16QAM symbols at a time interval has been used for studies. The channel is a fast fading channel which varies from each transmitted symbol vector to another one.

The BER performances of SD, original FSD, FSD without using the ordering and FSD with the simplified ordering are shown in Fig. 4. It can be seen that the BER performance of the FSD algorithm is very close to that of ML detection. The BER performance of FSD without using the ordering is also shown in Fig. 4. This algorithm is the same as the FSD algorithm except that the order for symbol detection is random. Comparing the BER performance with that of FSD shows that significant improvements in BER performance can be achieved by using the ordering. The BER of the FSD with our proposed simplified ordering is also shown in the same figure. It can be seen that the BER curve is nearly identical to that of the original FSD.

Figure 5 compares the complexity of FSD using the original and simplified orderings at different SNRs. The solid lines correspond to the average number of multiplication operations while the dashed lines correspond to the average number of addition operations. It can be seen that the simplified ordering reduces the complexity of FSD by more than $20 \%$. 


\section{CONCLUSIONS}

In this paper, we have analyzed the ordering of FSD from a geometrical point of view. Based on the analysis, we proposed a new ordering criterion to approximate the original ordering criterion for FSD. Computer simulation results have shown that the simplified ordering algorithm does not cause obvious degradation to the BER performance of the FSD, but reduces the complexity by more than $20 \%$.

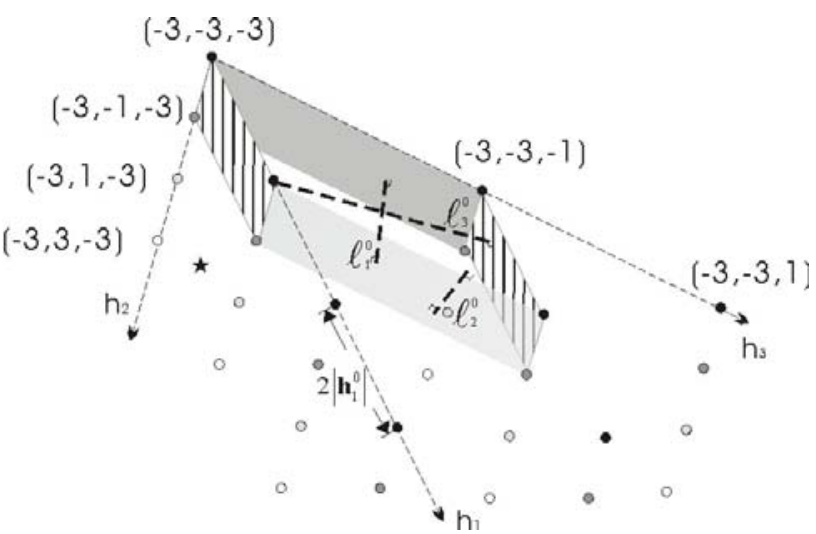

Fig. 1. Lattice in received signal space.

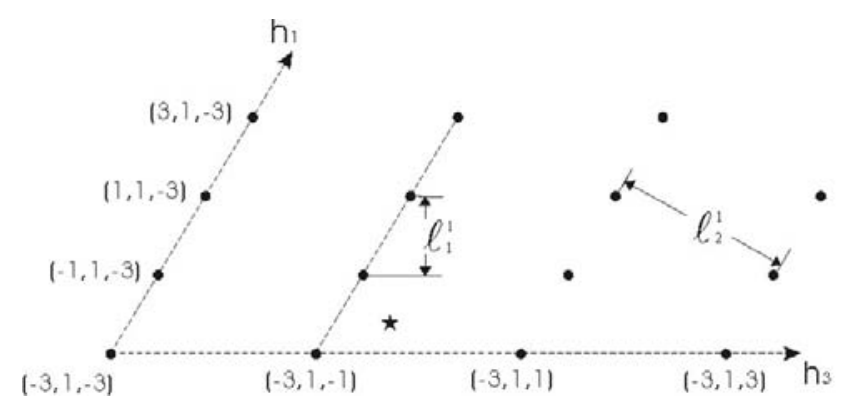

Fig. 2. Space spanned by $h_{1}$ and $h_{3}$.

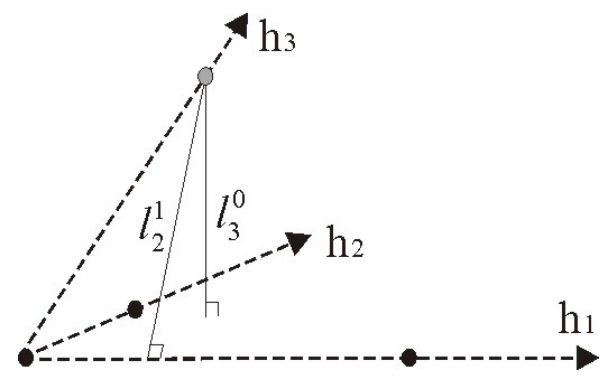

Fig. 3. Approximation of simplified ordering.

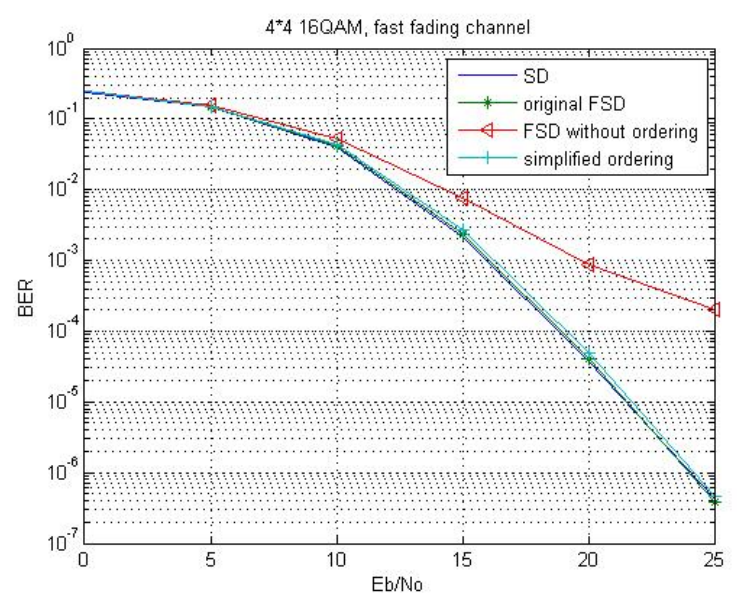

Fig. 4. Bit-error rate of FSD with and without simplified ordering.

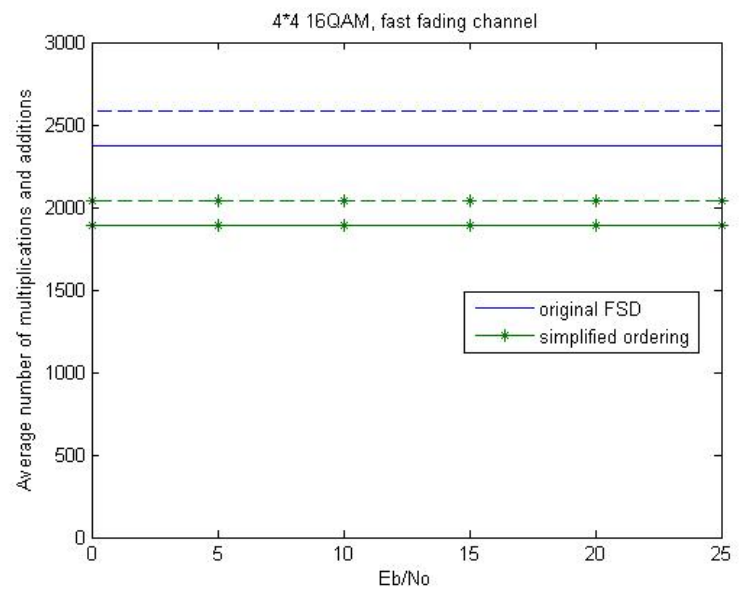

Fig. 5. Complexity of FSD with original and simplified ordering.

\section{REFERENCES}

[1] U. Fincke and M. Pohst, "Improved methods for calculating vectors of short length in lattice, including a complexity analysis”, Mathematics of Computation, vol. 44, no. 170, pp. 463-471, April, 1985.

[2] E. Viterbo and J. Boutros, "A universal lattice code decoder for fading channel”, IEEE Transactions on Information Theory, vol. 45,pp. 1639-1642, July 1999.

[3] E. Agrell, T. Eriksson, A. Vardy, and K. Zeger, "Closest point search in lattices”, IEEE Transactions on Information Theory, vol. 48, no. 8, pp. 2201-2214, August 2002.

[4] H. Artes, D. Seethaler and F. Hlawatsch, "Efficient detection algorithms for MIMO channels: a geometrical approach to approximate ML detection”, IEEE Transactions on Signal Processing, vol. 51, pp. 2808-2820, Nov. 2003.

[5] M. Samuel and M. P. Fitz, "Geometric Decoding Of PAM and QAM Lattices”, IEEE Global Telecommunications Conference GLOBECOM '07, pp. 4247-4252, Nov. 2007. 
[6] Z. Y. Shao, S. W. Cheung and T. I. Yuk, "A Simple and Optimum Geometric Decoding Algorithm for MIMO Systems", $4^{\text {th }}$

International Symposium on Wireless Pervasive Computing, ISWPC 2009, pp. 1-5, Feb. 2009.

[7] L. G. Barbero and J. S. Thompson, "Fixing the complexity of the sphere decoder for MIMO detection", IEEE Transactions on

Wireless Communications, vol. 7, no. 6, pp. 2131-2142, June 2008.
[8] J. Jalden, L. G. Barbero, B. Ottersten and J. S. Thompson, "The Error Probability of the Fixed-Complexity Sphere Decoder”, IEEE Transactions on Signal Processing, vol. 57, July, 2009.

[9] L. G. Barbero and J. S. Thompson, "Rapid prototyping of a fixedthroughput sphere decoder for MIMO systems," Proc. IEEE International Conference on Communications (ICC 06), vol. 7 , Istanbul, Turkey, June 2006, pp. 3082-3087. 